\title{
Selecting pharmacies for COVID-19 testing to ensure access
}

\author{
Simon Risanger ${ }^{1}$ (D) $\cdot$ Bismark Singh ${ }^{2} \cdot$ David Morton $^{3} \cdot$ Lauren Ancel Meyers ${ }^{4}$
}

Received: 30 July 2020 / Accepted: 30 November 2020 / Published online: 10 January 2021

(C) Springer Science+Business Media, LLC, part of Springer Nature 2021

\begin{abstract}
Rapid diagnostic testing for COVID-19 is key to guiding social distancing orders and containing emerging disease clusters by contact tracing and isolation. However, communities throughout the US do not yet have adequate access to tests. Pharmacies are already engaged in testing, but there is capacity to greatly increase coverage. Using a facility location optimization model and willingness-to-travel estimates from US National Household Travel Survey data, we find that if COVID-19 testing became available in all US pharmacies, an estimated $94 \%$ of the US population would be willing to travel to obtain a test, if warranted. Whereas the largest chain provides high coverage in densely populated states, like Massachusetts, Rhode Island, New Jersey, and Connecticut, independent pharmacies would be required for sufficient coverage in Montana, South Dakota, and Wyoming. If only 1,000 ZIP code areas for pharmacies in the US are selected to provide testing, judicious selection, using our optimization model, provides estimated access to 29 million more people than selecting pharmacies simply based on population density.
\end{abstract}

Keywords COVID-19 · Test access · Test site selection · Points of dispensing · Pharmacies · Facility location optimization

\section{Highlights}

- COVID-19 testing through pharmacies can improve access to communities across the US and simplify

Simon Risanger

simon.risanger@ntnu.no

Bismark Singh

bismark.singh@fau.de

David Morton

david.morton@northwestern.edu

Lauren Ancel Meyers

laurenmeyers@austin.utexas.edu

1 Department of Industrial Economics and Technology Management, Norwegian University of Science and Technology, Alfred Getz vei 3, 7491 Trondheim, Norway

2 Department of Mathematics, Friedrich-Alexander-Universität Erlangen-Nürnberg, Cauerstraße 11, 91058 Erlangen, Germany

3 Department of Industrial Engineering and Management Sciences, Northwestern University, 2145 Sheridan Road, Evanston, IL 60208, USA

4 Department of Integrative Biology and Department of Statistics and Data Sciences, The University of Texas at Austin, 1 University Station C0930, Austin, TX 78712, USA logistics. A data-driven optimization model judiciously recommends which pharmacies should offer testing.

- Pharmacies could provide access to an estimated 94\% of the US population, with coverage exceeding $80 \%$ in 47 states and $90 \%$ in 12 states.

- In densely populated states, a single large pharmacy chain could achieve broad coverage. Other states would require a combination of multiple chains, independent pharmacies, or alternative testing sites.

\section{Introduction}

Mass testing and contact tracing for COVID-19 will be critical to mitigate the impact of the disease prior to the availability of a vaccine [1,2]. While South Korea and Germany quickly launched expansive testing programs [3, 4], efforts are rolling out more slowly in the UK, Italy, and the US. In Guidelines for Opening Up America Again, the White House and the Centers for Disease Control and Prevention (CDC) provide benchmarks for states prior to relaxing COVID-19 social distancing orders [5]. A key criterion is "the ability to quickly set up safe and efficient screening and testing sites for symptomatic individuals and trace contacts of COVID+ results" [5]. Most states are expanding COVID-19 testing capacity at a diverse array of test sites, including commercial labs, healthcare 
clinics, hospitals, pharmacies, schools, churches, and sports complexes [6-8]. Although the US has increased its testing activity, fair access to testing remains a challenge. Data from New York City shows that people in poor and immigrant neighborhoods were less likely to be tested, despite having larger likelihood of testing positive [9]. According to the CDC, current data suggest that racial and ethnic minority groups have a disproportional burden of illness [10].

There are two classes of SARS-CoV-2 tests: viral tests that assay for current infection and antibody tests that detect prior infection. Viral testing allows for contact tracing and case isolation strategies to contain emerging clusters of cases [11]. Individuals infected with SARS-CoV-2 are thought to be infectious several days before they feel symptoms [12]. Thus, wide testing of all who believe they may be at risk for infection can improve pandemic mitigation [13]. However, when testing capacity is limited, individuals with COVID-like symptoms or known exposure are prioritized $[14,15]$.

A state or federally coordinated effort to offer testing through pharmacies could rapidly address gaps in coverage in the US. Pharmacies are ubiquitous and well positioned to expand access to COVID-19 testing. Many people feel more comfortable walking into a pharmacy than a local hospital or outpatient clinic, and many live closer to a pharmacy than a healthcare facility [16, 17], with over $90 \%$ of Americans living within five miles of a pharmacy [16]. Pharmacies have a workforce skilled to administer tests [4]. As of 2017, there were an estimated 2,800 retail walk-in clinics in the US, with three quarters operated by CVS or Walgreens [18]. Large pharmacy chains have extensive distribution networks, which were leveraged during the $2009 \mathrm{H} 1 \mathrm{~N} 1$ pandemic by several states to distribute federally allocated antivirals [19]. Finally, the Association of State and Territorial Health Officials (ASTHO) recognizes pharmacies as instrumental for immunization campaigns and administering vaccines [19]. Given the vital role pharmacies could play in rapidly expanding COVID-19 testing capacity and accessibility, the US Department of Health \& Human Services (HHS) approved pharmacies to order and administer tests for COVID-19 as of April 8, 2020 [20].

Pharmacies are already engaged in testing. HHS has established a public-private partnership with selected pharmacies and retailers. Their goal is to provide more accessible and convenient testing and to ensure protection of healthcare personnel [21]. As of July 2020, selected locations from CVS, Kroger, Rite Aid, Walgreens, Walmart, and independent pharmacies in partnership with Health Mart and eTrueNorth, are offering SARS-CoV-2 tests [21]. Pharmacies are also scaling up their own testing beyond the HHS-sponsored community-based testing sites. CVS and Walgreens offer drive-through testing in several states, with the intention of expanding testing locations [22, 23].
Expansion of pharmacy-based COVID-19 testing would not only help bridge existing gaps between current capacity and national reopening targets, but also provide access for hard-to-reach and at-risk communities. The focus of this paper is to estimate the geographic coverage that could be achieved through a pharmacy-based testing program, in terms of the proportion of individuals who would be willing to travel to the nearest pharmacy testing site to obtain a COVID-19 test (if warranted), and the distance they would be required to travel. To accomplish this, we use an optimization model to identify specific pharmacy locations in a given state or nationwide that would provide the greatest access. Our findings indicate whether and where major chains can provide sufficient access on their own.

\section{Methods}

Facility location problems have wide application in healthcare systems, spanning non-emergency location of hospitals, clinics, blood banks, and laboratories, and location of emergency centers, ambulance stations, and temporary medical facilities; see, e.g., the survey by Ahmadi-Javid et al. [24] for an overview. Facility location for administering COVID-19 testing can be classified as selecting points of dispensing, which differs from traditional health care facility location problems because a key concern is to provide equitable and easy access to all [24]. Depending on application and focus, the points-of-dispensing problem and solution methods take different forms in the literature, including combining simulation and optimization [25], queueing approximations for drive-through dispensing [26], and addressing demand uncertainty through chance constraints [27]. Our objective is to estimate access to selected COVID-19 test sites, and corresponding travel distances, on national and state levels in the US. The resulting problem instances become large in scale. Computational and data availability considerations therefore limit a detailed representation of the system. We adopt a model by Singh et al. [28], which optimizes pharmacy-based distribution of antiviral drugs for Texas for the $2009 \mathrm{H} 1 \mathrm{~N} 1$ pandemic. The model is relatively simple to solve and intuitive, and is available as a Web-based decision support tool for the Texas Department of State Health Services [29].

The model requires as input a geographic region - e.g., a US state - partitioned into ZIP code areas. Given a specific number of locations (ZIP code areas) to be selected, solving the optimization model yields the subset of store locations that maximizes the expected number of people willing to travel to their closest pharmacy, among the selected locations. That is, the optimization model maximizes coverage of the population given a limited "budget" of locations, which can be selected. We solve the optimization 
model parametrically in this budget to understand the tradeoff between the total number of locations and coverage.

Mathematically, we represent the problem as the facility location optimization model expressed in formulation (1). Table 1 outlines the nomenclature. The objective (1a) is to maximize the population willing to travel to its closest selected pharmacy. Index $i \in I$ denotes all areas (ZIP code areas), while $j \in J \subseteq I$ denotes areas with pharmacies. The population willing to travel from area $i$ to its closest selected pharmacy is the product of the population of area $i, P_{i}$, the fraction of population willing to travel from area $i$ to $j, \rho_{i j}$, and binary variable $y_{i j}$, which takes value one if area $j$ contains the closest selected pharmacy for area $i$ and zero otherwise. Constraint (1b) sets the budget of locations, where $N$ is a parameter and variable $x_{j}$ expresses whether a pharmacy in area $j$ is selected. Constraint (1c) ensures that each area $i$ can only choose one pharmacy area $j$ as its closest. Note that because the model can only select one $y_{i j}=1$ for each area $i$, it chooses the $y_{i j}$ that maximizes objective (1a). Constraint (1d) ensures that an area $i$ cannot choose a pharmacy in area $j$ if it has not been selected. Finally, Eqs. 1e and $1 \mathrm{f}$ force $x_{j}$ and $y_{i j}$ to be binary.

The model requires as input the population's willingness to travel. We use the data-driven willingness-to-travel function from [28], which is estimated using National Household Travel Survey data [30] for privately operated vehicles. Equation 2 shows the fraction of the population, $\rho$, willing to travel distance $d$. The willingness-totravel function is an exponentially decaying fraction of the population, where the population has different travel behavior for distances below and above a fivemile threshold. This threshold considers different mobility patterns in urban and rural areas, where the willingness to travel has a steeper decrease below five miles. We calculate parameter $\rho_{i j}$ as $\rho\left(d_{i j}\right)$ where $d_{i j}$ is the distance between areas $i$ and $j$.

$$
\begin{aligned}
\max _{x, y} & \sum_{i \in I} \sum_{j \in J} P_{i} \rho_{i j} y_{i j} \\
\text { s.t. } & \sum_{j \in J} x_{j} \leq N \\
& \sum_{j \in J} y_{i j} \leq 1 \quad \forall i \in I \\
& y_{i j} \leq x_{j} \quad \forall i \in I, j \in J \\
& x_{j} \in\{0,1\} \quad \forall j \in J \\
& y_{i j} \in\{0,1\} \quad \forall i \in I, j \in J \\
\rho(d)= \begin{cases}e^{-0.109 d^{1.184}}, \\
1.479 e^{-0.4255 d^{0.6025}}, & \text { if } d<5\end{cases} & \text { otherwise. }
\end{aligned}
$$

Table 1 Nomenclature for optimization problem (1)

$\begin{array}{ll}\begin{array}{ll}\text { Sets and indices } \\ i \in I\end{array} & \text { Set of all areas } \\ j \in J \subseteq I & \text { Set of areas that contain a pharmacy } \\ \begin{array}{ll}\text { Parameters } & \text { Population of area } i \\ P_{i} & \text { Fraction of population willing to travel } \\ \rho_{i j} & \text { from area } i \text { to area } j\end{array} \\ \begin{array}{ll}\text { Mecision variables } \\ x_{j} \in\{0,1\} & \text { Indicates area } j \text { is selected for testing site(s) } \\ y_{i j} \in\{0,1\} & \text { Indicates closest selected testing area is } j \text { for area } i\end{array}\end{array}$

For areas, we use a mapping from ZIP codes to ZIP Code Tabulation Areas (ZCTAs). The former represents a set of mail delivery routes, while ZCTAs correspond to a geographic area used by the US Census Bureau. Population counts, centroid coordinates, and land areas for each ZCTA are obtained from the 2010 US Census. The US Census provides coordinates, latitudes and longitudes, for the centroid of each ZCTA. We calculate travel distance $d_{i j}$ as the distance between two ZCTA centroid coordinates, as calculated by the Haversine formula. As in Singh et al. [28], we assume that the full population in a ZCTA is willing to travel to a pharmacy in their own ZCTA. The travel distance to pharmacies within a ZCTA is approximated as the square root of land area of the ZCTA divided by two for the full population. For pharmacies, we use a national dataset for operating pharmacies in the US, produced by InfoGroup and Amazon Web Services in coordination with Esri's national COVID-19 response team.

Note that the optimization model only considers whether one or more pharmacies is present in a particular ZCTA. Hence, the model does not consider the capacity of each pharmacy to provide diagnostic testing. That said, local pharmacy capacity (supply) is arguably naturally sized to be proportional to demand by market forces. Our data indicate the number, but not the size, of individual pharmacies in each ZCTA, and we analyze the strong positive correlation between this number and the size of the local population in Section 4. In effect, our model assumes a pharmacy located at the centroid of each ZCTA, but in reality selecting an area, $j$, that is densely populated amounts to selecting multiple pharmacies in that ZCTA.

Formulation (1) requires a decision variable $y_{i j}$ between each ZCTA and pharmacy location. This is computationally burdensome for model instances for large states and the nation. A full national run has over 477 million pairs, while large states like California and Texas each have over 1.9 million pairs. Each pair becomes a decision variable 
in the model. To reduce computational burden, we only include variables for combinations that have at least a $20 \%$ willingness to travel. A post-processing step connects ZCTAs with less than a $20 \%$ willingness to travel to their closest pharmacy. The $20 \%$ cut-off balances computational time and performance. National runs now have $420,702 y_{i j}$ variables, while California and Texas instances have 47,078 and 21,590 pairs, respectively. We implement formulation (1) in Julia, using the open source package JuMP [31]. The code is freely available via https://github.com/srisanger/ selecting-pharmacies-for-COVID-testing. Table 2 outlines computational results using Gurobi version 9.0.2 on a laptop with an Intel i7-8550U processor for different values of $N$ on a diverse subset of states. Even though we use a cutoff at $20 \%$ willingness to travel, this approximation has small impact on the resulting objective function values and significantly reduces computation time.

\section{Results}

Our analysis considers 67,473 pharmacies within the US. CVS, Walgreens, and Walmart are the three largest chains, and operate $15.1 \%, 13.0 \%$, and $6.9 \%$ of these stores, respectively. We consider two scenarios: a nation-wide program administered federally and state-wide programs administered separately by the 50 states.

We estimate that a national pharmacy-based testing program using the entire network of major chains and independent stores would provide access for $94.0 \%$ of the US population. Section A in the online supplement provides detailed results. Collectively, all pharmacies, both chains and independents, operate stores in 14,474 unique ZCTAs nationwide, which is less than half of the 32,930 ZCTAs in the US Census. The resulting median distance from home to the nearest pharmacy-based COVID19 testing site would be 2.4 miles, with $90 \%$ of the population living within 7.4 miles of the nearest testing site.

Reducing the number of pharmacy test sites would reduce access, but not by much if the pharmacy locations were specifically selected to ensure broad access. For example, 10,000 geographically dispersed stores - chosen via the optimization model to maximize willingness to travel - would be expected to provide access to $90.3 \%$ of the US population, with a 2.5 -mile median travel distance, and $90 \%$ of people living within 8.0 miles of the nearest COVID-19 testing site. Half as many pharmacy test sites (located in 5,000 ZCTAs) would reduce expected access to $77.7 \%$ while increasing the median and the 90th percentile travel distance to 3.1 miles and 10.8 miles, respectively. Figures 1 and 2 show how estimated access grows and travel distances shrink as the number of testing sites grows for national model instances.

To simplify coordination and logistics, a policymaker could rely on a single major chain. Although none of the major chains can reach over $80 \%$ of the US population, Table 3 shows that the median travel distance to the nearest test site would be expected to remain under five miles. Any pair of the major chains - CVS, Walgreens, and Walmart - could provide $80 \%$ access. CVS and Walgreens could reach an estimated $84.2 \%$ of the population; adding Walmart
Table 2 Comparison of results for full model and model with willingness-to-travel cut-off at $20 \%$

\begin{tabular}{llll}
\hline & Population coverage [\%] & & \\
& $25 \%$ of all & $50 \%$ of all & $75 \%$ of all \\
& pharmacies & pharmacies & pharmacies \\
New Jersey & $82.51(82.51)$ & $92.64(92.64)$ & $97.00(97.00)$ \\
California & $76.21(76.20)$ & $89.36(89.35)$ & $95.51(95.51)$ \\
Texas & $70.28(70.22)$ & $84.86(84.83)$ & $92.22(92.20)$ \\
Colorado & $69.82(69.79)$ & $83.91(83.87)$ & $91.24(91.24)$ \\
Wyoming & $50.19(50.03)$ & $77.02(77.02)$ & $86.14(86.03)$ \\
& Computation time $[\mathrm{s}]$ & & \\
& $25 \%$ of all & $50 \%$ of all & $75 \%$ of all \\
pharmacies & pharmacies & pharmacies \\
New Jersey & $13.4(0.7)$ & $7.4(0.5)$ & $7.6(0.4)$ \\
California & $370.5(1.8)$ & $152.5(1.3)$ & $113.1(1.0)$ \\
Texas & $361.3(1.0)$ & $144.6(0.3)$ & $134.3(0.3)$ \\
Colorado & $3.4(0.1)$ & $2.4(0.1)$ & $2.4(0.1)$ \\
Wyoming & $0.1(0.02)$ & $0.1(<0.01)$ & $0.1(<0.01)$ \\
\hline
\end{tabular}

Results for the model with willingness-to-travel cut-off at $20 \%$ are in parentheses for both objective function value (top half of table) and computation time (bottom half) in seconds 
Fig. 1 Estimated percent of US population willing to travel to its closest selected pharmacy with a growing number of ZCTAs that have pharmacies to administer COVID-19 tests, i.e., with parameter $N$ growing in model (1). Results are for all pharmacies and for individual pharmacy chains

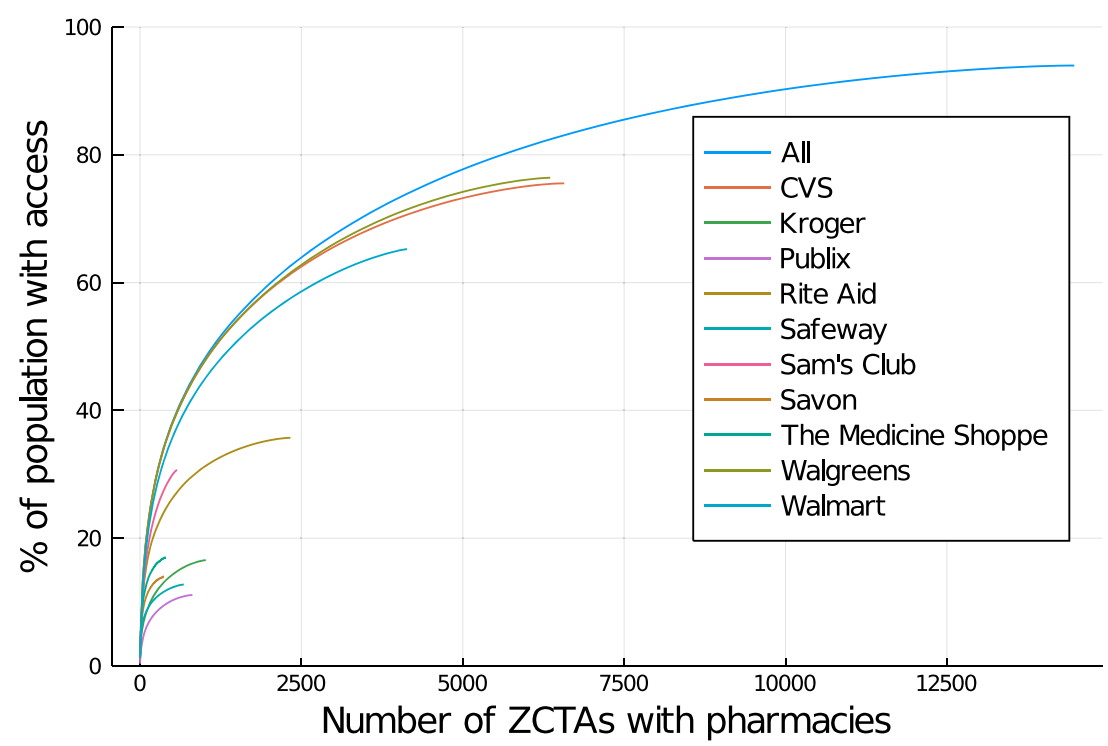

would slightly expand coverage to $86.5 \%$, but still fall short of the $94.0 \%$ estimated if all US pharmacies offer testing.

We also analyzed the coverage of state-level pharmacy testing sites. See Section B in the online supplement for details. If all chain and independent pharmacies provide COVID-19 testing, we estimate that 47 states would achieve at least $80 \%$ access. Only Vermont (77.3\%), Alaska (78.0\%), and Maine (79.9\%) fall short. Access is estimated to exceed $95 \%$ in 12 states, including the most populous states of California (97.8\%), Florida (97.3\%), New York (95.8\%), and Texas $(95.0 \%)$.

If states were to offer testing through just a single chain, we estimate that CVS, Walgreens, and Walmart stores could reach over $80 \%$ of the population in 17,13 , and 7 US states, respectively. Single chains can provide far greater access to COVID-19 testing in densely populated states than in sparsely populated states. For example, in Massachusetts, Rhode Island, New Jersey, and Connecticut, CVS - the largest pharmacy chain in these states - achieves over $80 \%$ access, with half of the population traveling no more than 2.3 miles to their nearest CVS store. In contrast, the largest pharmacy chain in Montana, South Dakota, and Wyoming would be expected to provide accessible testing to under $50 \%$ of the respective populations, with half living over 9.0 miles and $10 \%$ living over 50 miles from the nearest store in the chain.
Fig. 2 Distances the US population must travel (median, 10th percentile, and 90th percentile) under an increasing number of selected ZCTAs that have pharmacies to administer tests, based on results from model (1)

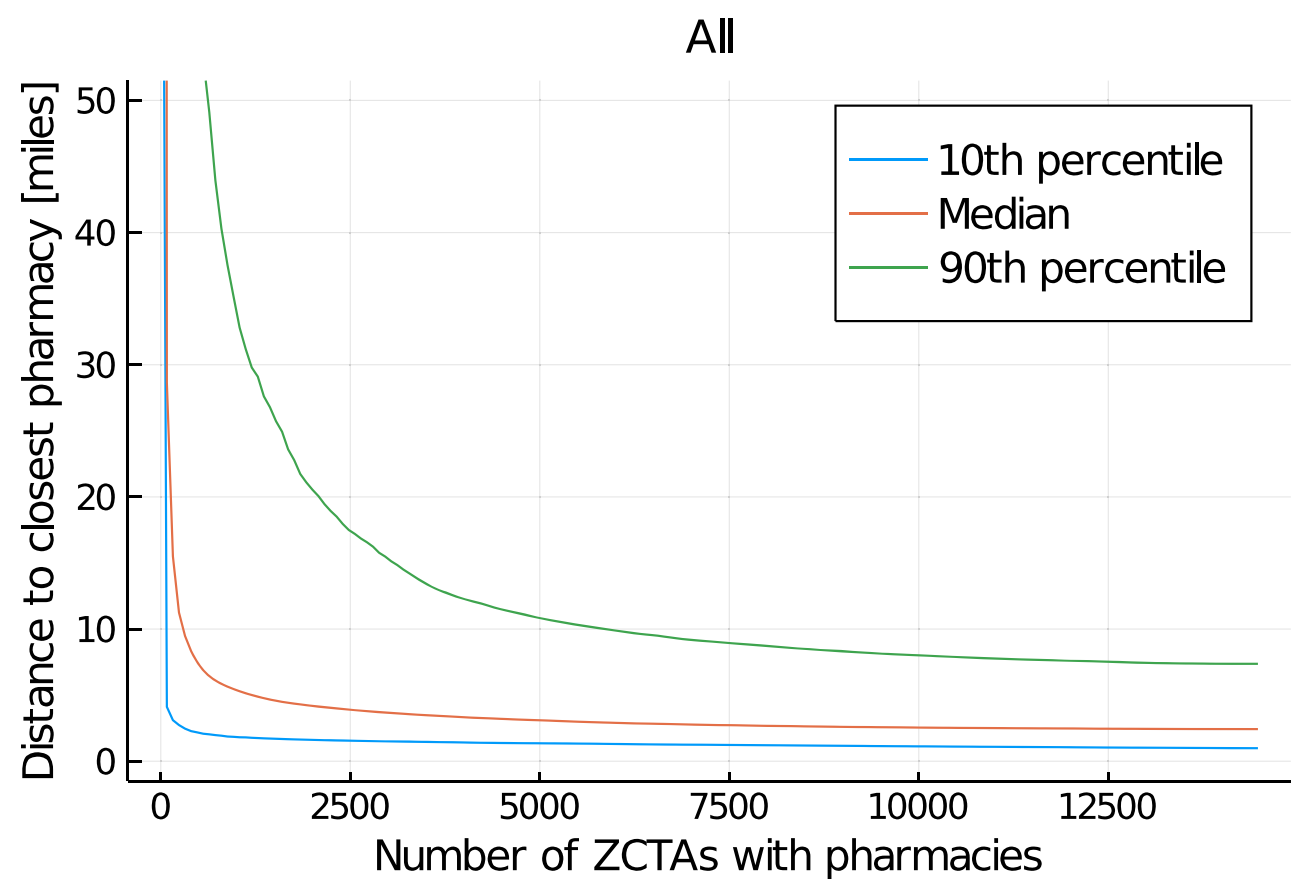


Table 3 Estimated proportion of the US population willing to travel to the nearest pharmacy and corresponding travel distances

\begin{tabular}{lllll}
\hline & Population & \multicolumn{2}{l}{ Travel distance [miles] } \\
\cline { 3 - 5 } Chain & access & 10th percentile & Median & 90th percentile \\
\hline All & $94.0 \%$ & 1.0 & 2.4 & 7.4 \\
All (max 10,000 locations) & $90.3 \%$ & 1.1 & 2.5 & 8.0 \\
All (max 5,000 locations) & $77.7 \%$ & 1.4 & 3.1 & 10.8 \\
CVS & $75.5 \%$ & 1.1 & 2.9 & 15.4 \\
Walgreens & $76.4 \%$ & 1.2 & 3.0 & 13.5 \\
Walmart & $65.2 \%$ & 1.8 & 4.5 & 12.1 \\
CVS \& Walgreens & $84.2 \%$ & 1.0 & 2.6 & 10.4 \\
CVS \& Walmart & $82.6 \%$ & 1.1 & 2.8 & 10.1 \\
Walgreens \& Walmart & $81.8 \%$ & 1.2 & 2.8 & 10.2 \\
CVS, Walgreens \& Walmart & $86.5 \%$ & 1.0 & 2.6 & 9.3 \\
\hline
\end{tabular}

As of July 2020, there is not a comprehensive listing of US testing sites. However, Georgia, Illinois, and Minnesota provide state-level lists [6-8]. Table 4 outlines the results. With locations per 28 July 2020, we estimate that testing locations in Illinois and Minnesota are accessible to slightly above $70 \%$ of the state populations, while Georgia's sites cover just over $50 \%$ of the population. If testing were offered at all chain and independent pharmacies, all three states can significantly increase access and reduce travel distance. The largest pharmacy chain in Georgia and Illinois would be expected to provide better coverage than current sites, especially in Georgia where CVS stores could provide access to over $80 \%$ of the population. All three states could significantly increase their access by adding the locations of the largest pharmacy chain to their current testing sites.

\section{Discussion}

As US states ramp up COVID-19 testing to allow broad and fair access, our results suggest that pharmacy-based testing would provide good coverage and reasonable travel distances for large portions of the US population. We provide an actionable strategy for selecting specific pharmacy test sites to ensure access, given that the density and proximity of pharmacies varies enormously across the US. We have applied the strategy to demonstrate the potential reach of national-level and state-level pharmacy testing programs. Our approach can likewise be used to select pharmacies on a local scale or to close known gaps in coverage by ongoing testing efforts.

During the 2009 H1N1 influenza pandemic, pharmacies worked with state health departments to distribute strategic
Table 4 Estimated access in three states from existing test sites (per 28 July 2020) [6-8] and pharmacies (all and largest chain) with travel distances

\begin{tabular}{|c|c|c|c|c|c|}
\hline \multirow[b]{2}{*}{ State } & \multirow[b]{2}{*}{ Distribution } & \multirow{2}{*}{$\begin{array}{l}\text { Population } \\
\text { access }\end{array}$} & \multicolumn{3}{|c|}{ Travel distance [miles] } \\
\hline & & & 10th percentile & Median & 90th percentile \\
\hline \multirow[t]{4}{*}{ Georgia } & All pharmacies & $94.6 \%$ & 1.8 & 3.4 & 7.4 \\
\hline & Existing test sites & $53.5 \%$ & 2.8 & 6.5 & 13.3 \\
\hline & CVS & $80.5 \%$ & 1.8 & 3.7 & 10.7 \\
\hline & CVS \& existing & $86.1 \%$ & 1.7 & 3.6 & 8.4 \\
\hline \multirow[t]{4}{*}{ Illinois } & All pharmacies & $94.9 \%$ & 0.9 & 1.8 & 5.8 \\
\hline & Existing test sites & $73.5 \%$ & 1.0 & 2.6 & 10.7 \\
\hline & Walgreens & $86.8 \%$ & 0.9 & 1.9 & 9.1 \\
\hline & Walgreens \& existing & $88.8 \%$ & 0.9 & 1.8 & 7.4 \\
\hline \multirow[t]{4}{*}{ Minnesota } & All pharmacies & $89.5 \%$ & 1.1 & 3.4 & 9.0 \\
\hline & Existing test sites & $73.7 \%$ & 1.3 & 4.4 & 12.3 \\
\hline & Walgreens & $70.8 \%$ & 1.2 & 3.9 & 23.5 \\
\hline & Walgreens \& existing & $81.8 \%$ & 1.2 & 3.9 & 11.3 \\
\hline
\end{tabular}


national stockpile antivirals [19]. Our findings suggest that tailoring testing strategies to individual states could achieve broader coverage than a one-size-fits-all national strategy. Densely populated states, like Rhode Island, Massachusetts, and New York may be able to simplify testing logistics by coordinating only with a single chain. In less densely populated states, like Montana, South Dakota, and Wyoming, enlisting more than a single pharmacy chain would be required to reach more than half of the population.

For the majority of states that fall between these two extremes, judicious selection of pharmacy locations is important but not obvious. Our optimization approach would help such states to provide the broadest possible access to COVID-19 testing while keeping the coordination simple. Consider, for instance, Illinois and Texas. Both have densely populated metropolitan areas and significant rural populations. Our analysis suggests a viable strategy that would work well for both. First, identify the major chain with the best geographic reach - Walgreens provides an estimated $86.8 \%$ coverage in Illinois and CVS provides an estimated $80.6 \%$ coverage in Texas - and then selectively target single independent pharmacies to cover the remainder of the largely rural population. An additional 50 independent pharmacies in Illinois and 182 in Texas would be expected to raise coverage to $90 \%$.

Our approach is simple and effective. As a benchmark, consider an alternative in which pharmacies are selected as testing sites based simply on local population density without considering travel distances. If we select testing sites in only 1,000 ZCTAs, then this heuristic would choose pharmacies primarily in dense urban areas and thereby provide access to an estimated $39.3 \%$ of the US population. In contrast, our approach identifies pharmacies that would be expected to increase coverage to $48.1 \%$, and hence provide access to an estimated additional 29 million people. For 5,000 pharmacies, our approach again beats this heuristic, raising expected coverage from $73.9 \%$ to $77.7 \%$ of the US population, a difference of 12.5 million people. The benefits of our pharmacy selection strategy are amplified when resources are scarce and coverage is limited.

We estimate that, as of 28 July 2020, COVID-19 test sites in Georgia barely provide access for half of the state population and test sites in Illinois and Minnesota miss over a quarter of their populations. Georgia reports 150 test sites, and if they were to provision COVID-19 tests through all 2,345 pharmacies in the state (or alternatively, one in each of the 424 ZCTAs that have a pharmacy), estimated access would increase from $53.5 \%$ to $94.6 \%$ of the population. Offering testing through the single largest pharmacy chain in the state (CVS) in addition to current sites would increase estimated coverage to $86.1 \%$. Ramping up testing in Walgreens stores in Illinois and Minnesota would also narrow the gap in coverage, but to a lesser extent.

We make a critical assumption that may bias our estimates of people's willingness to travel to nearby pharmacies for COVID-19 test when symptoms present. Using data from the National Household Travel Survey [30], we assume that individual willingness to travel for diagnostic testing coincides with willingness to travel by privately operated vehicle for work, school, family or social reasons under normal, non-emergency, conditions. Our estimates may be pessimistic, given the urgency of COVID-19, or possibly optimistic, if COVID-19 symptoms reduce willingness to leave the home. Still, surveys suggest that the US population is willing to seek COVID-19 tests. In one such survey, $70 \%$ claim that they are willing to take a free test [32], while $80 \%$ are willing to pay less than $\$ 2$ for at-home antibody test kits [33]. If our estimates are incorrect but in a consistent manner across the US population - for example, if willingness to travel is higher than we estimate by a consistent proportion - then the estimated coverage will be similarly incorrect, but the optimal selection of pharmacies would remain the same.

We also note that our analysis considers the location (ZCTA) but not the capacity of each pharmacy. As outlined in Section 2, the optimization problem does not distinguish single versus multiple pharmacies in a ZCTA. Commercial pharmacy capacity may be sized to handle local demand under normal operations but may not suffice to handle a surge in demand. Nevertheless, policymakers can open additional pharmacies or other sites in high demand areas if they experience insufficient capacity. The data show a strong correlation between population and pharmacies at a ZCTA (Pearson correlation coefficient of 0.819), which suggests that there are more pharmacies in areas of high demand. However, if situations arise where policymakers are unable to open additional pharmacies in a particular ZCTA and require pharmacies outside that area, it will affect the willingness to travel and hence the coverage. We also note that the supply of test kits and the processing capacity can be limiting factors, and we do not consider these in our analysis.

Our pharmacy selection methodology can flexibly accommodate plausible policy constraints. For example, we can identify specific stores within chains that have already launched testing programs to expand access to under-served populations. If states have established targets for accessibility of COVID-19 testing, we can determine the minimum number and best locations of pharmacies required to achieve such targets. When recommending additional pharmacy testing sites, the method can account for existing COVID-19 testing capacity through pharmacies, labs, healthcare systems, public health centers and other sites. 
It can also exclude chains or individual stores from consideration, such as pharmacies co-located with grocery stores to reduce exposure to healthy shoppers, or pharmacies without adequate facilities or staff.

Willingness-to-travel estimates can provide insight into geographic and socioeconomic disparities in access to diagnostic tests and other pharmacy-provided resources during the COVID-19 pandemic. For instance, Fouad et al. [34] show that COVID-19 affects African Americans disproportionately and recommends ensuring equitable testing, while Tsai and Wilson [35] explain how COVID-19 can be a public health problem for homeless populations. Our approach can rapidly identify candidate pharmacybased testing sites to ameliorate such gaps.

\section{Conclusion}

Although testing capacity has been increasing since February 2020, many communities still lack adequate access to COVID-19 tests in the US. The bottleneck is not just the quantity of tests, and the ability to process tests, but also the number, staffing and locations of test sites. To meet these needs, pharmacies have selectively begun to ramp up COVID-19 testing. Our results suggest that broad and coordinated pharmacy-based testing programs can provide estimated access to $94 \%$ of the US population. Even a subset of pharmacies, for instance pharmacies of a specific chain, could ameliorate critical gaps in coverage. The facility location optimization is a simple method for selecting locations to improve access, which can adjust to policy constraints. For instance, densely populated states can provide acceptable access through a single pharmacy chain, while other states can use the optimization framework to judiciously select locations to maximize cover. As America continues to 'open up', robust and expansive testing, contact tracing, and isolation programs will be key to mitigating future transmission and averting unmanageable surges in COVID-19 hospitalizations. Our procedure for determining testing sites can be easily applied to fill in persistent geographic and socioeconomic gaps in coverage across the US.

\footnotetext{
Acknowledgments The authors thank Dr. Gordon Wells for acquiring and sharing the pharmacy data set used in this study, and for providing additional pharmacy data sets that we considered. The authors are also grateful to John Sheffield and Mauricio Tec, who helped gather and share pharmacy data sets. We acknowledge financial support from the National Institutes of Health under Grant NIH R01 AI151176 and Grant NIH U01 GM087791, the U.S. Department of Homeland Security under Grant 2017-ST-061-QA0001 and Tito's Handmade Vodka. The views and conclusions contained in this document are those of the authors and should not be interpreted as necessarily representing the official policies, either expressed or implied, of the U.S. Department of Homeland Security.
}

Funding National Institutes of Health grants NIH R01 AI151176 and NIH U01 GM087791, U.S. Department of Homeland Security grant 2017-ST-061-QA0001 and Tito's Handmade Vodka.

\section{Compliance with Ethical Standards}

Conflict of interests The authors declare no competing interest.

Supplementary Information The online version contains supplementary material available at (10.1007/s10729-020-09538-w).

\section{References}

1. Peto J (2020) Covid-19 mass testing facilities could end the epidemic rapidly. BMJ 368:m1163. https://doi.org/10.1136/bmj. m1163

2. World Health Organization: Coronavirus disease 2019 (COVID19): situation report, 72. Tech. rep. (2020). https://apps.who.int/ iris/bitstream/handle/10665/331685/nCoVsitrep01Apr2020-eng. pdf. Accessed 16 Nov 2020

3. Cohen J, Kupferschmidt K (2020) Countries test tactics in war against COVID-19. Science 367(6484):1287-1288. https://doi. org/10.1126/science.367.6484.1287

4. Chen SW (2020) US pharmacists can now test for coronavirus they could do more if government allowed it. The Conversation. https://theconversation.com/us-pharmacists-can-now-test-for-coro navirus-they-could-do-more-if-government-allowed-it-135188. Accessed 16 Nov 2020

5. The White House: Opening Up America Again (2020). https:// www.whitehouse.gov/openingamerica/. Accessed 16 Nov 2020

6. Georgia Department of Public Health: Find a Covid 19 Testing Site (2020). https://dph.georgia.gov/locations/covid-19-testingsite. Accessed 28 Jul 2020

7. Illinois Department of Public Health: COVID-19 Testing Sites (2020). https://dph.illinois.gov/covid19/covid-19-testing-sites. Accessed 28 Jul 2020

8. Minnesota COVID-19 response: Find Testing Locations in Minnesota (2020). https://mn.gov/covid19/for-minnesotans/if-sick/ testing-locations/index.jsp\#header. Accessed 28 Jul 2020

9. Borjas GJ (2020) Demographic determinants of testing incidence and COVID-19 infections in new york city neighborhoods. SSRN Electronic Journal. https://doi.org/10.2139/ssrn.3572329

10. Centers for Disease Control and Prevention: COVID-19 in Racial and Ethnic Minority Groups (2020). https://www.cdc.gov/corona virus/2019-ncov/need-extra-precautions/racial-ethnic-minorities.html. Accessed 16 Nov 2020

11. Hellewell J, Abbott S, Gimma A, Bosse NI, Jarvis CI, Russell TW, Munday JD, Kucharski AJ, Edmunds WJ, Sun F, Flasche S, Quilty BJ, Davies N, Liu Y, Clifford S, Klepac P, Jit M, Diamond C, Gibbs H, van Zandvoort K, Funk S, Eggo RM (2020) Feasibility of controlling COVID-19 outbreaks by isolation of cases and contacts. The Lancet Global Health 8(4):e488-e496. https://doi.org/10.1016/S2214-109X(20)30074-7

12. Du Z, Xu X, Wu Y, Wang L, Cowling BJ, Meyers LA (2020) Serial interval of COVID-19 among publicly reported confirmed cases. Emerging Infectious Disease Journal 26(6):1341. https://doi.org/10.3201/eid2606.200357

13. Patel R, Babady E, Theel ES, Storch GA, Pinsky BA, George KS, Smith TC, Bertuzzi S (2020) Report from the American Society for Microbiology COVID-19 International Summit, 23 March 2020: Value of Diagnostic Testing for SARS-cov-2/COVID-19. mBio 11(2). https://doi.org/10.1128/mbio.00722-20 
14. Fenton NE, Neil M, Osman M, McLachlan S (2020) COVID-19 infection and death rates: the need to incorporate causal explanations for the data and avoid bias in testing. Journal of Risk Research 23(7-8):862-865. https://doi.org/10.1080/13669877. 2020.1756381

15. Spinelli A, Pellino G (2020) COVID-19 pandemic: perspectives on an unfolding crisis. British Journal of Surgery. https://doi.org/ 10.1002/bjs.11627

16. Broughel J, Yatsyshina Y (2020) Relax pharmacy regulations to help with COVID-19 testing and treatment. SSRN Electronic Journal. https://doi.org/10.2139/ssrn.3564463

17. Mason $P$ (2004) Near-patient testing in the pharmacy. Pharmaceutical Journal 272:708-710

18. RAND Corporation: The Evolving Role of Retail Clinics (2016). https://doi.org/10.7249/RB9491-2

19. Association of State and Territorial Health Officials: Pharmacy Legal Toolkit (2014). https://www.astho.org/Infectious-Disease/ Pharmacy-Legal-Toolkit/. Accessed 16 Nov 2020

20. U.S. Department of Health \& Human Services: HHS Statements on Authorizing Licensed Pharmacists to Order and Administer COVID-19 Tests (2020). https://www.hhs.gov/about/news/2020/ 04/08/hhs-statements-on-authorizing-licensed-pharmacists-to-orderand-administer-covid-19-tests.html. Accessed 16 Nov 2020

21. US Department of Health \& Human Services: CommunityBased Testing Sites for COVID-19 (2020). https://www.hhs.gov/ coronavirus/community-based-testing-sites/index.html. Accessed 16 Nov 2020

22. CVS Health: COVID-19 testing: Information and locations (2020). https://cvshealth.com/covid-19/testing-information-locati on-locations. Accessed 16 Nov 2020

23. Walgreens: Drive-thru COVID-19 Testing at Walgreens (2020). https://www.walgreens.com/findcare/covid19/testing. Accessed 16 Nov 2020

24. Ahmadi-Javid A, Seyedi P, Syam SS (2017) A survey of healthcare facility location. Computers \& Operations Research 79:223-263. https://doi.org/10.1016/j.cor.2016.05.018. http:// www.sciencedirect.com/science/article/pii/S0305054816301253

25. Lee EK, Chen CH, Pietz F, Benecke B (2009) Modeling and optimizing the public-health infrastructure for emergency response. INFORMS Journal on Applied Analytics 39(5):476490. https://doi.org/10.1287/inte.1090.0463
26. Ramirez-Nafarrate A, Lyon JD, Fowler JW, Araz OM (2015) Point-of-dispensing location and capacity optimization via a decision support system. Production and Operations Management 24(8):1311-1328. https://doi.org/10.1111/poms.12323. https://onlinelibrary.wiley.com/doi/abs/10.1111/poms.12323

27. Murali P, Ordóñez F, Dessouky MM (2012) Facility location under demand uncertainty: response to a large-scale bio-terror attack. Socio-Economic Planning Sciences 46(1):7887. https://doi.org/10.1016/j.seps.2011.09.001. http://www. sciencedirect.com/science/article/pii/S0038012111000450. Special Issue: Disaster Planning and Logistics: Part 1

28. Singh B, Huang HC, Morton DP, Johnson GP, Gutfraind A, Galvani AP, Clements B, Meyers LA (2015) Optimizing distribution of pandemic influenza antiviral drugs. Emerg Infect Dis 21(2):251-258. https://doi.org/10.3201/eid2102.141024

29. Meyers LA (2020) Texas Pandemic Flu Toolkit. https://flu.tacc. utexas.edu/. Accessed 16 Nov 2020

30. U.S. Department of Transportation: Data Extraction Tool and Visualization Prototypes (2009). https://nhts.ornl.gov/det/default. aspx. Accessed 16 Nov 2020

31. Dunning I, Huchette J, Lubin M (2017) Jump: a modeling language for mathematical optimization. SIAM Rev 59(2):295320. https://doi.org/10.1137/15M1020575

32. Thunström L, Ashworth M, Shogren JF, Newbold S, Finnoff D (2020) Testing for COVID-19: willful ignorance or selfless behavior? Behavioural Public Policy: 1-18. https://doi.org/10.1017/bpp. 2020.15

33. Serra-Garcia M, Szech N (2020) Demand for COVID-19 antibody testing, and why it should be free. SSRN Electronic Journal. https://doi.org/10.2139/ssrn.3607484

34. Fouad MN, Ruffin J, Vickers SM (2020) COVID-19 Is out of proportion in african americans. This will come as no surprise.. The American Journal of Medicine. https://doi.org/10.1016/j.amjmed. 2020.04.008

35. Tsai J, Wilson M (2020) COVID-19: a potential public health problem for homeless populations. The Lancet Public Health 5(4):e186-e187. https://doi.org/10.1016/S2468-2667(20)30053-0

Publisher's note Springer Nature remains neutral with regard to jurisdictional claims in published maps and institutional affiliations. 